Volume 03 Number 02 Maret 2020

\title{
HIGH-LEVEL MODELING OF BUILDING STRUCTURE USING OCTOGONAL CASTELLATED BEAM AND STEEL PLATE SHEAR WALL SYSTEM (SPSW)
}

\author{
Ridwan Dwi Setiawan \\ Civil Engineering Study Program \\ Narotama University Surabaya \\ Arif Rahman Hakim 51 Street, Sukolilo, Surabaya \\ rdsetiawan.21@gmail.com \\ Koespiadi \\ Civil Engineering Study Program \\ Narotama University Surabaya \\ Arif Rahman Hakim 51 Street, Sukolilo, Surabaya \\ koespiadi@narotama.ac.id
}

\begin{abstract}
In reality, the development of a city or region is realized in the form of high-rise buildings. In the world of construction usually planner or executor of a project generally choose between two such material is steel or concrete. Concrete is a construction material that forms most often for current construction projects. In this thesis is exemplified how to choose the steel material in construction. steel profile is quite popular to use the castellated profile, by adding a steel plate shear wall (steel plate shear wall). The result of this structural modeling for building a dental hospital in Malang, get the dimensions of the roof joists with spans7.2 $\mathrm{m}$ on the roof using WF profile $300 \times 150 \times 6.5 \times 9$ and on the floor with the original profile WF 300 $\times 200 \times 8 \times 14$, then converted into a castellated beam profile octogonal $442.5 \times 200 \times 8 \times 14$. The dimensions of the transverse roof beam using a profile castellated beam octogonal 427.5 $\times 200 \times 8 \times 12$ and longitudinal roof beam using octogonal profile castellated beam $570 \times 200$ $x 8 \times 13$. b. Dimensions of the transverse floor beam profile using a profile octogonal castellated beam $427.5 \times 200 \times 8 \times 12$ and beam elongated floor using octogonal profile castellated beam $617.5 \times 300 \times 10 \times 15$. The dimensions of the column using a 1-4 floor King Cross profile $588 \times 300 \times 12 \times 20$, the dimensions of the floor columns 5-7 using a profile King Cross $450 \times 200 \times 9 \times 14$.
\end{abstract}

Keywords :Building Structure, Modeling, Castellated Beam, Steel Plate Shear Wall.

\section{INTRODUCTION}

Rise buildings constructed based on the limited use of land, especially in urban areas is also high demand for space for different types of activities. Humans possible to build increasingly high-rise buildings. State building higher and higher, allowing it to require strength in the planning structure. A planner need a material in its construction. The material often used of concrete or steel. Steel material at this time many variations, including a fairly popular use is castellated profile. Castellated profile has various types of openings are hexagonal, octagonal 
IJTI (International Journal Of Transparantion Infrastructure) Available Online @http://jurnal.narotama.ac.id/index.php/ijti ISSN: 2597-4769 (ONLINE) ISSN : 2597-4734 (CETAK)

Volume 03 Number 02 Maret 2020

and round. In this thesis, we planned a multi-story building using octagonal castellated beams and paired a steel plate shear walls.

From the above background, this thesis has several formulation issues such as:

1. How to determine a preliminary design castellated steel beam cross-sectional profile?

2. How to plan a secondary structure include joist, floor plate and the roof and the stairs?

3. How to calculate the load acting on the structure of the building?

\section{Castellated Steel Profile}

\section{LITERATURE REVIEW}

Castellated steel profile is a solid profile (profile WF) which is split into two equal parts in a straight line along the span, with a certain pattern in accordance with the shape of openings to be created. The shape of the openings were varied from a hexagon (hexagonal), segidelapan (octogonal), or rounded (circullar) wherein the shape and size of openings customized needs. (Hutabarat and Syahrizal, 2017)

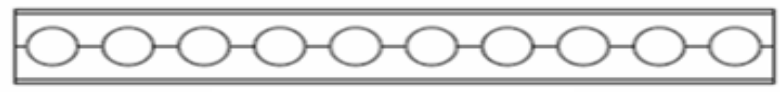

(a) Circular web opening

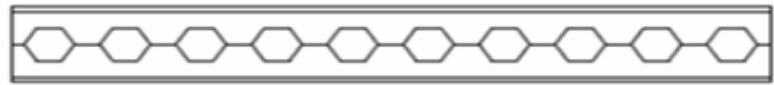

(b) Hexagonal web opening

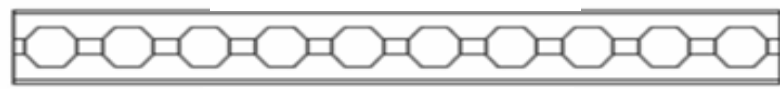

(c) Octogonal web opening

Figure 1. Castellated with various openings

\section{Steel Plate Shear Wall}

Steel plate shear wall is a lateral load resisting system consisting of a solid vertical steel plates connecting beams and columns around which installed along the height of the structure so as to form a buttress. (Seilie and Hooper, 2005)

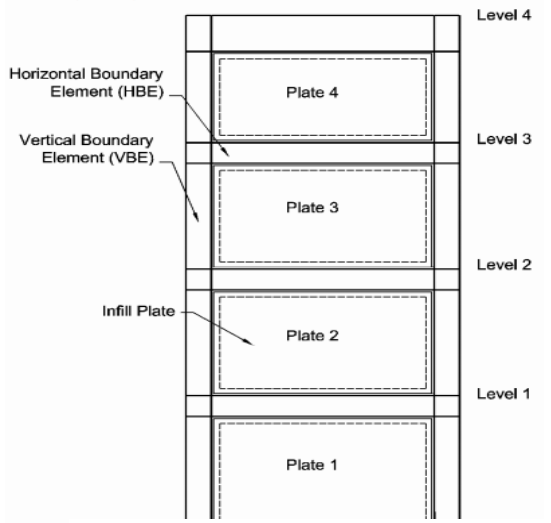

Figure 2. Steel Plates Shear Wall

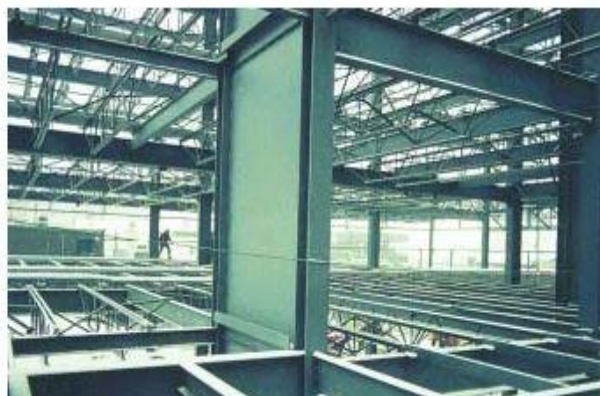

Figure 3. Model of Steel Plates Shear Wall 


\section{RESEARCH METHODS}

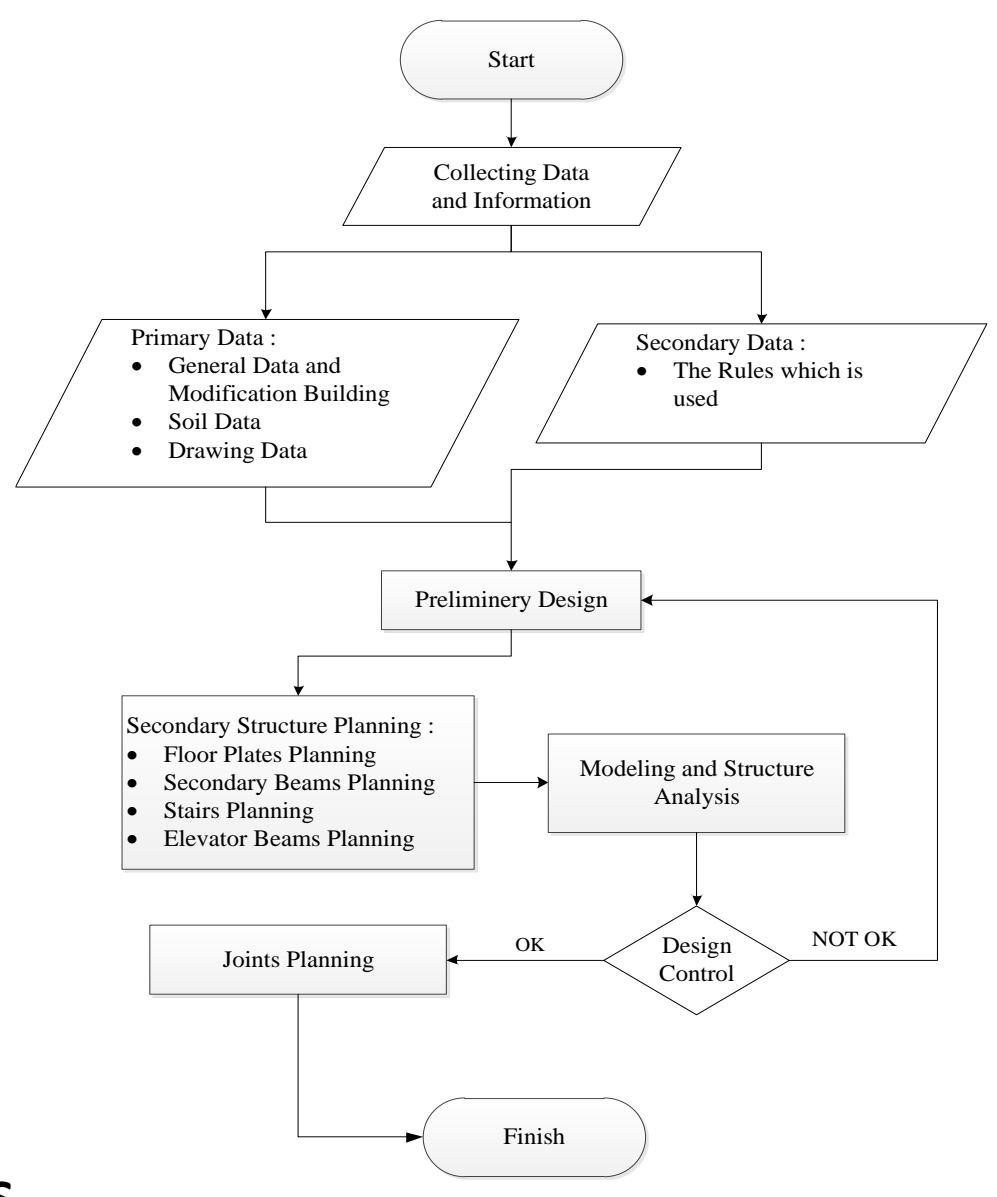

Figure 4. Flow Chart Method Final Settlement

\section{Collecting Data}

\section{RESULTS AND DISCUSSION}

\section{General Data Building}

$\begin{array}{ll}\text { Building names } & \text { : Dental Hospital of Brawijaya University. } \\ \text { Building location } & \text { : Veteran Street, Malang, East Java } \\ \text { Building functions } & \text { : Hospital. } \\ \text { Floors } & : 7 \text { floors. } \\ \text { Building height } & : \text { 29, 35 meters. } \\ \text { Main structure } & : \text { Conventional Concrete }\end{array}$

Modification Data Building
Building names
: Dental Hospital of Brawijaya University.
Building location
: Veteran Street, Malang, East Java.
Building functions
: Hospital.
Floors
: 7 floors.
Building height
: 29, 35 meters.
Main structure
: Profiles Octogonal Castellated Beam and King Cross Column.
Structure System
: Steel Plate Shear Wall. 
IJTI (International Journal Of Transparantion Infrastructure) Available Online @http://jurnal.narotama.ac.id/index.php/ijti ISSN: 2597-4769 (ONLINE) ISSN : 2597-4734 (CETAK)

Volume 03 Number 02 Maret 2020

\section{Secondary planning}

- Plates Planning

On the roof and floor plate is planned to use floor deck with $0.75 \mathrm{~mm}$ thickness and quality of concrete (fc): $25 \mathrm{Mpa}$. Having its own weight of $10.1 \mathrm{~kg} . \mathrm{m} 2$ concrete plates.

Table 1. Table of Plates Planning

\begin{tabular}{cccccc}
\hline Plates & $\begin{array}{c}\text { Useful } \\
\text { load } \\
\text { Element }\end{array}$ & $\begin{array}{c}\text { Span } \\
(\mathrm{kg})\end{array}$ & $\begin{array}{c}\text { Plates } \\
\text { Thick } \\
(\mathrm{m} 2)\end{array}$ & $\begin{array}{c}\text { Negative } \\
\text { Reinforcement } \\
(\mathrm{cm} 2 / \mathrm{m})\end{array}$ & Reinforcement \\
\hline Roof & 200 & 8 & 9 & 1.968 & $\emptyset 10-200$ \\
Floor & 500 & 8 & 9 & 2,199 & $\varnothing 12-250$ \\
\hline
\end{tabular}

- Secondary Beams Planning

On the secondary beams are planned using WF profile on the roof and octagonal castellated on the floor plate of the building.

Table 2. Table of Secondary Beams

\begin{tabular}{cccccc}
\hline $\begin{array}{c}\text { Element of } \\
\text { Beam }\end{array}$ & Type & \multicolumn{2}{c}{$\begin{array}{c}\text { Interaction } \\
\text { Equation } \\
\text { (requirement } \\
<1.0)\end{array}$} & $\begin{array}{c}\mathrm{f} \\
\text { (permission) }\end{array}$ & $\mathrm{f}$ (max) \\
\hline Roof & WF 300.200.8.12 & 0.54 & OK & $2 \mathrm{~cm}$ & $1,85 \mathrm{~cm}$ \\
Floor & $442,5.200 .8 .14$ & 0.78 & OK & $2 \mathrm{~cm}$ & $1,25 \mathrm{~cm}$ \\
& & & & & 0.39 \\
Elevator & WF 400.200.8.13 & 0.24 & OK & $1 \mathrm{~cm}$ & $\mathrm{~cm}$ \\
\hline
\end{tabular}

- Stairs Planning

Here are the results obtained for planning the steps of:

Table 3. Table of Stairs Planning

\begin{tabular}{|c|c|c|c|c|c|}
\hline \multirow{2}{*}{ Element } & \multirow{2}{*}{ Type } & \multicolumn{2}{|c|}{$\mathrm{Mu}(\mathrm{kg} \mathrm{m})$} & \multicolumn{2}{|c|}{ Deflection } \\
\hline & & $\mathrm{Mu}$ & $\varnothing M n$ & $f^{\prime}$ & $f^{\circ}$ \\
\hline Stairs & $\begin{array}{c}4 \mathrm{~mm} \text { steel } \\
\text { plate }\end{array}$ & 17.05 & 139.5 & $\begin{array}{c}0.08 \\
3\end{array}$ & 0.052 \\
\hline $\begin{array}{l}\text { Rung } \\
\text { Bracing }\end{array}$ & L 65.65.8 & $\begin{array}{c}117.3 \\
9\end{array}$ & $\begin{array}{c}179.3 \\
3\end{array}$ & $0: 43$ & 0.32 \\
\hline $\begin{array}{c}\text { Stairs } \\
\text { Landing }\end{array}$ & $\begin{array}{c}\text { Plates Steel } 9 \\
\mathrm{~mm}\end{array}$ & $\begin{array}{c}489.6 \\
3\end{array}$ & $\begin{array}{c}706.2 \\
2\end{array}$ & 0.47 & 0.32 \\
\hline $\begin{array}{l}\text { Beams of } \\
\text { Stairs } \\
\text { Landing }\end{array}$ & $\begin{array}{c}\text { WF } \\
100.50 .5 .7\end{array}$ & $\begin{array}{c}450.1 \\
12\end{array}$ & 945 & 0.43 & 0.2 \\
\hline & & & & 1.23 & 0.80 \\
\hline
\end{tabular}


IJTI (International Journal Of Transparantion Infrastructure) Available Online @http://jurnal.narotama.ac.id/index.php/ijti ISSN: 2597-4769 (ONLINE) ISSN : 2597-4734 (CETAK)

\begin{tabular}{cccccc}
\multicolumn{7}{c}{ Volume 03 Number 02 Maret 2020 } \\
\hline Main & WF & 2973. & 6862. & & \\
Beam & 250.125 .5 .8 & 33 & 5 & & \\
Stairs & & & & & \\
Fulcrum & WF & 4731. & 6862. & 1 & 0.86 \\
Beam & 250.125 .5 .8 & 24 & 5 & & \\
Stairs & & & & &
\end{tabular}

\section{Control Structure Design and Modeling}

In modeling the structure using the SAP 2000 program assistance, then controlled by restriction analysis results according to the rules SNI 03-1726-2012 restrictions on earthquake resilience planning procedures for building and non-building structures of buildings. The goal is that the system is being planned structure becomes more feasible.

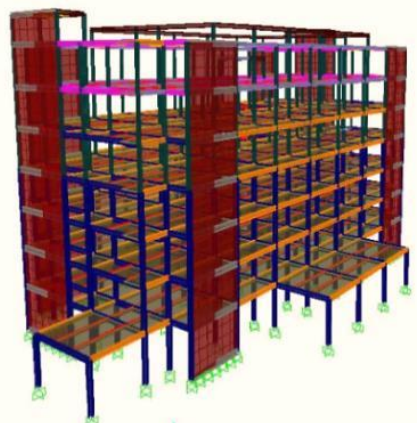

Figure 5. Structure of Building Modeling Planned

- Mass Participation Control

Control of mass participation should include sufficient amount of variance in order to get the mass participation of at least $90 \%$ of the actual mass in the horizontal direction orthogonal to the response to be reviewed.

Table 4. Ratio Mass Participation

\begin{tabular}{ccrcrr}
\hline OutputCase & StepType & StepNum & Period & SumUX & SumUY \\
\hline text & text & Unitless & Sec & unitless & unitless \\
MODAL & Mode & 18 & 0.26432 & 0742 & 0919 \\
MODAL & Mode & 19 & 0.21806 & 0919 & 0919 \\
\hline
\end{tabular}

- Natural Shakes Time Control Fundamentals

Control of natural shakes fundamental time (Ta) in seconds, can be determined by the following formula equation:

$\mathrm{Ta}=\mathrm{Ct} . \mathrm{hn}^{\mathrm{x}}$

$\mathrm{Ct}=0,0488$

$\mathrm{x}=0,75$

$\mathrm{hn}=29.4 \mathrm{~m}$

$\mathrm{Ta}=0.0488 \times 29,40,75=0.616 \mathrm{sec}$

SD1 value $=0.383$, with $\mathrm{Cu}=1.4$ obtained from table rules SNI 03-1726-2012

So the structures that permitted period; 
IJTI (International Journal Of Transparantion Infrastructure) Available Online @http://jurnal.narotama.ac.id/index.php/ijti ISSN: 2597-4769 (ONLINE) ISSN : 2597-4734 (CETAK)

Volume 03 Number 02 Maret 2020

$\mathrm{T}=\mathrm{Ta} . \mathrm{Cu}=0.616 .1 .4=0.8626 \mathrm{sec}$

Table 5. Period and Frequency Building Structure

\begin{tabular}{clrcc}
$\begin{array}{c}\text { OutputCase } \\
\text { Text }\end{array}$ & $\begin{array}{c}\text { StepType } \\
\text { text }\end{array}$ & $\begin{array}{c}\text { StepNum } \\
\text { unitless }\end{array}$ & $\begin{array}{c}\text { Period } \\
\text { Sec }\end{array}$ & $\begin{array}{c}\text { Frequency } \\
\text { Cyc / sec }\end{array}$ \\
\hline MODAL & Mode & 1 & 0.811471 & 1.232329506 \\
MODAL & Mode & 2 & 0.811471 & 1.232330644 \\
MODAL & Mode & 3 & 0.753497 & 1.327144532 \\
MODAL & Mode & 4 & 0.739337 & 1.352562521 \\
MODAL & Mode & 5 & 0.738095 & 1.354838833 \\
& & & & \\
MODAL & Mode & 23 & 0.092806 & 10.77520708 \\
MODAL & Mode & 24 & 0.089164 & 11.21526172 \\
\hline
\end{tabular}

- End Value Response Spectrum Control

Based on ISO 1726-2012, the final value of the dynamic response of building structures in the specified direction can not be less than $85 \%$ of the value of the static response or (Vynamic $\geq 0.85$ Vstatic).

Table 6. Base Reaction of Structure Building

\begin{tabular}{lccc}
\hline Output case & $\begin{array}{c}\text { V dynamic } \\
(\mathrm{kg})\end{array}$ & V static $(\mathrm{kg})$ & $\begin{array}{c}\text { V dynamic } \geq \mathrm{V} \\
\text { static }\end{array}$ \\
\hline Earthquake & $758,067.93$ & & OK \\
$\begin{array}{l}\text { Directions } X \\
\text { Earthquake }\end{array}$ & $758,226.65$ & $758,066.887$ & \\
Directions $Y$ & & OK \\
\hline
\end{tabular}

- Control deviation (drift)

Calculation of the deviation between the floor $(\Delta)$ is calculated in order to distinguish the deflection at the center of mass at the top and bottom are reviewed.

Table 7. Inter-Floor Control Deviation Charges Due Earthquake Directions $X$

\begin{tabular}{|c|c|c|c|c|c|c|c|}
\hline Floor & $\begin{array}{c}\text { hi } \\
\text { (Mm) }\end{array}$ & $\begin{array}{c}\delta x e \\
(\mathrm{Mm})\end{array}$ & $\begin{array}{c}\Delta \mathrm{x} \\
(\mathrm{Mm})\end{array}$ & $\begin{array}{c}\Delta=\delta x a- \\
\delta x b \\
(\mathrm{Mm})\end{array}$ & $\begin{array}{c}\Delta \mathrm{a} \\
(\mathrm{Mm})\end{array}$ & $\begin{array}{l}\Delta \mathrm{a} / \rho \\
(\mathrm{Mm})\end{array}$ & $\begin{array}{c}\Delta \\
<\Delta \mathrm{a} \\
/ \rho\end{array}$ \\
\hline $\begin{array}{c}1 \\
\text { (Basic) }\end{array}$ & 0 & 0 & & 0 & 0 & 0 & OK \\
\hline 2 & 4200 & 3,585 & $\begin{array}{c}14 \\
341 \\
33\end{array}$ & 14341 & 63 & 48 & OK \\
\hline 3 & 4200 & $\begin{array}{c}8442 \\
13\end{array}$ & $\begin{array}{c}769 \\
55\end{array}$ & 19428 & 63 & 48 & OK \\
\hline 4 & 4200 & $\begin{array}{c}889 \\
19\end{array}$ & $\begin{array}{c}557 \\
78\end{array}$ & 21788 & 63 & 48 & OK \\
\hline 5 & 4200 & 501 & 004 & 22447 & 63 & 48 & OK \\
\hline
\end{tabular}


IJTI (International Journal Of Transparantion Infrastructure) Available Online @http://jurnal.narotama.ac.id/index.php/ijti ISSN: 2597-4769 (ONLINE) ISSN : 2597-4734 (CETAK)

\begin{tabular}{ccccccccc}
\multicolumn{8}{c}{ Volume 03 Number 02 Maret 2020 } \\
\hline 6 & 4200 & 550 & 200 & 24196 & 63 & 48 & OK \\
& & 31 & 124 & & & & \\
7 & 4200 & 060 & 239 & 22039 & 63 & 48 & OK \\
& & 35 & 141 & & & & \\
8 & 4200 & 269 & 077 & 16839 & 63 & 48 & OK \\
roof & & 36 & 144 & & & & \\
beams & 1800 & 157 & 628 & 3,551 & 27 & 21 & OK \\
& & 36 & 145 & & & & \\
Upstairs & 1100 & 329 & 315 & 0687 & 17 & 13 & OK \\
\hline
\end{tabular}

Table 8. Inter-Floor Control deviation Expenses Due to Earthquake Directions $Y$

\begin{tabular}{|c|c|c|c|c|c|c|c|}
\hline Floor & $\begin{array}{c}\text { hi } \\
(\mathrm{Mm})\end{array}$ & $\begin{array}{c}\delta x e \\
(\mathrm{Mm})\end{array}$ & $\begin{array}{c}\Delta \mathrm{x} \\
(\mathrm{Mm})\end{array}$ & $\begin{array}{c}\Delta=\delta x a- \\
\delta x b \\
(\mathrm{Mm})\end{array}$ & $\begin{array}{c}\Delta \mathrm{a} \\
(\mathrm{Mm})\end{array}$ & $\begin{array}{l}\Delta \mathrm{a} / \rho \\
(\mathrm{Mm})\end{array}$ & $\begin{array}{c}\Delta \\
<\Delta \mathrm{a} \\
/ \rho\end{array}$ \\
\hline $\begin{array}{c}1 \\
\text { (Basic) }\end{array}$ & 0 & 0 & 0 & 0 & 0 & 0 & OK \\
\hline 2 & 4200 & 4,424 & $\begin{array}{c}17 \\
695 \\
39\end{array}$ & 17695 & 63 & 48 & OK \\
\hline 3 & 4200 & 9834 & $\begin{array}{c}337 \\
61\end{array}$ & 21642 & 63 & 48 & OK \\
\hline 4 & 4200 & 15409 & $\begin{array}{c}635 \\
82\end{array}$ & 22298 & 63 & 48 & OK \\
\hline 5 & 4200 & 20595 & $\begin{array}{l}380 \\
110\end{array}$ & 20745 & 63 & 48 & OK \\
\hline 6 & 4200 & 27,500 & $\begin{array}{l}001 \\
133\end{array}$ & 27622 & 63 & 48 & OK \\
\hline 7 & 4200 & 33482 & $\begin{array}{l}927 \\
148\end{array}$ & 23925 & 63 & 48 & OK \\
\hline $\begin{array}{c}8 \\
\text { roof }\end{array}$ & 4200 & 37007 & $\begin{array}{l}027 \\
153\end{array}$ & 14101 & 63 & 48 & OK \\
\hline beams & 1800 & 38410 & $\begin{array}{l}642 \\
161\end{array}$ & 5,614 & 27 & 21 & OK \\
\hline Upstairs & 1100 & 40358 & 430 & 7788 & 17 & 13 & OK \\
\hline
\end{tabular}

4. Primary Structure Planning and Connections

- Primary Structure Planning

In the calculation and planning of the building to the main structure used octogonal castellated beams on the lengthwise and crosswise direction and the column used kingcross profile. 
IJTI (International Journal Of Transparantion Infrastructure) Available Online @http://jurnal.narotama.ac.id/index.php/ijti ISSN: 2597-4769 (ONLINE) ISSN : 2597-4734 (CETAK)

Volume 03 Number 02 Maret 2020

Table 9. Calculation of Main Beams Recap

\begin{tabular}{|c|c|c|c|c|c|c|}
\hline $\begin{array}{l}\text { Type } \\
\text { column }\end{array}$ & $\mathrm{m}$ & $\begin{array}{l}\text { Dimension } \\
\qquad \mathrm{Mm}\end{array}$ & $\begin{array}{c}\text { Strong } \\
\text { Bending } \\
\text { Moment } \\
\varnothing \mathrm{Mn}<\mathrm{Mu} \\
(\mathrm{kg} . \mathrm{m})\end{array}$ & $\begin{array}{l}\text { Strong } \\
\text { Slide } \\
\varnothing \vee n<\mathrm{Vu} \\
\text { (Kg.m) }\end{array}$ & $\begin{array}{c}\text { Deflectio } \\
\qquad \\
f^{\prime}>f^{\circ}\end{array}$ & $\begin{array}{l}\text { Interacti } \\
\text { on } \\
\text { Equation }\end{array}$ \\
\hline CB 1 & 8 & $\begin{array}{c}\text { CB. } 617,5 x \\
300 \times 10 \times 15\end{array}$ & $\begin{array}{c}75622.06 \\
<28091.39\end{array}$ & $\begin{array}{c}23224.12 \\
<14592.98\end{array}$ & $\begin{array}{c}2.22 \\
<0.57\end{array}$ & $0.36>1$ \\
\hline CB 2 & 8 & $\begin{array}{c}\text { CB. } 580 \times 20 \\
0 \times 8 \times 13\end{array}$ & $\begin{array}{l}43452.72 \\
<4350.70\end{array}$ & $\begin{array}{l}17287.06 \\
<2797.74\end{array}$ & $\begin{array}{c}2.22 \\
<0.01\end{array}$ & $0.80>1$ \\
\hline CB 1a & 7.2 & $\begin{array}{l}\text { CB. } 427,5 x \\
200 \times 8 \times 12 \\
\end{array}$ & $\begin{array}{c}27835.35 \\
<14251.89\end{array}$ & $\begin{array}{l}12223.86 \\
<9942.44\end{array}$ & $2<0.48$ & $0.01>1$ \\
\hline
\end{tabular}

Table 10. Calculation Column Recap

\begin{tabular}{|c|c|c|c|c|c|c|c|}
\hline \multirow{2}{*}{$\begin{array}{l}\text { Type } \\
\text { column }\end{array}$} & \multirow[b]{2}{*}{$\mathrm{m}$} & \multirow{2}{*}{$\begin{array}{l}\text { Dimension } \\
\text { Mm }\end{array}$} & \multicolumn{3}{|c|}{ Analysis Structure } & \multirow{2}{*}{$\begin{array}{c}\text { Nominal } \\
\text { Moment } \\
\text { Control } \\
\varnothing \mathrm{Mn}< \\
\mathrm{Mu} \\
\text { (kg.m) }\end{array}$} & \multirow{2}{*}{$\begin{array}{l}\text { Interacti } \\
\text { on } \\
\text { Equation }\end{array}$} \\
\hline & & & Pu (kg) & $\begin{array}{l}\text { Mux } \\
(\mathrm{kg} \mathrm{m})\end{array}$ & $\begin{array}{l}\text { Muy } \\
\text { (Kg.m) }\end{array}$ & & \\
\hline $\begin{array}{l}\text { Column } \\
\text { It. 1-4 }\end{array}$ & 4.2 & $\begin{array}{c}\text { KC. } 588 \times 30 \\
0 \times 12 \times 20\end{array}$ & $\begin{array}{c}291,983 . \\
99\end{array}$ & $\begin{array}{c}152.7 \\
3\end{array}$ & $\begin{array}{c}10816 . \\
51\end{array}$ & $\begin{array}{c}97209> \\
152.73\end{array}$ & $0.927>1$ \\
\hline $\begin{array}{l}\text { Column } \\
\text { It. 5-7 }\end{array}$ & 4.2 & $\begin{array}{c}\text { KC. } 450 \times 20 \\
0 \times 9 \times 14\end{array}$ & $\begin{array}{c}106,350 . \\
31\end{array}$ & $\begin{array}{c}311.3 \\
2\end{array}$ & $\begin{array}{c}8462.0 \\
8\end{array}$ & $\begin{array}{c}35370> \\
311.32\end{array}$ & $0.912>1$ \\
\hline
\end{tabular}

- Steel Plate Shear Wall Planning

SAP2000 analysis results obtained from the shear force on a steel plate shear wall that is:

$\mathbf{V}_{\mathbf{u}}=53497,85 \mathrm{~kg}$

Using a steel material BJ 37

fy $=2400 \mathrm{~kg} / \mathrm{cm}^{2}$ and

$\mathrm{fu}=3700 \mathrm{~kg} / \mathrm{cm}^{2}$

Dimension of Steel Plate Shear Wall $=3.6 \mathrm{mx} 2.1 \mathrm{~m}$

Steel Plate Shear Wall Thickness

Assumptions of SPSW pull angle of $30^{\circ}$ from the VBE.

$\varnothing \mathrm{V}_{\mathrm{n}} \quad=0.90 \times 0: 42 \times \mathrm{f}_{\mathrm{y}} \times \mathrm{xx} \sin (2) \mathrm{t}_{\mathrm{w}} \mathrm{L}_{\mathrm{cf}} \alpha$

$53497.85=0.90 \times 0: 42 \times 2400$ pixels $\mathrm{t}_{\mathrm{w}} \times 360 \times \sin \left(2 \times 30^{\circ}\right)$

$\mathrm{t}_{\mathrm{w}} \quad=0.189 \mathrm{~cm}$, then use $\mathrm{t}_{\mathrm{w} \text { min }}=0.3 \mathrm{~cm}$

Pull angle Actual accordance thickness SPSW

$$
\begin{aligned}
& \alpha=\tan ^{-1} \sqrt[4]{\frac{1+\frac{t L}{2 A_{c}}}{1+\operatorname{thn}\left[\frac{1}{A_{b}}+\frac{h^{3}}{360 I_{c} L}\right]}} \\
& \alpha \quad=\tan ^{-1} \sqrt[4]{\frac{1+\frac{0,3 \times 360}{2 \times 385}}{1+0,3 \times 210\left[\frac{1}{84,12}+\frac{210^{3}}{360 \times 127020 \times 360}\right]}}
\end{aligned}
$$


IJTI (International Journal Of Transparantion Infrastructure) Available Online @http://jurnal.narotama.ac.id/index.php/ijti ISSN: 2597-4769 (ONLINE) ISSN : 2597-4734 (CETAK)

Volume 03 Number 02 Maret 2020

$\alpha \quad=4180^{\circ}$ qualify $30^{\circ}<\alpha<55^{\circ}$

Scroll strength Actual SPSW

$$
\begin{aligned}
& \emptyset V_{n}=0.90 \times 0: 42 \times f_{y} \times x \times \sin (2) t_{w} L_{c f} \alpha \\
& \varnothing V_{n}=0.90 \times 0: 42 \times 2400 \times 03 \times 360 \times \sin \left(2 \times 41.80^{\circ}\right) \\
& \varnothing V_{n}=97366,79 \mathrm{~kg}>53497.85 \mathrm{~kg} \ldots . \text { (OK) }
\end{aligned}
$$

- Joint Planning

Joint with the planned use bolts of quality:

Profile BJ 41, $\mathrm{fu}=410 \mathrm{MPa}=4100 \mathrm{~kg} / \mathrm{cm}^{2}$

\begin{tabular}{|c|c|c|c|c|}
\hline \multicolumn{2}{|c|}{ Element } & $\begin{array}{l}\text { Elbow } \\
\text { Profile }\end{array}$ & $\begin{array}{c}\mathrm{D} \\
(\mathrm{mm})\end{array}$ & $\begin{array}{c}n \\
\text { (bh) }\end{array}$ \\
\hline $\begin{array}{l}\text { Secondary } \\
\text { Floors }\end{array}$ & $\begin{array}{l}\text { Secondary } \\
\text { Beams body }\end{array}$ & L60.60.6 & 12 & 3 \\
\hline $\begin{array}{l}\text { Beam - } \\
\text { Main Beam } \\
\text { joint }\end{array}$ & $\begin{array}{l}\text { Main Beams } \\
\text { body }\end{array}$ & L60.60.6 & 12 & 3 \\
\hline $\begin{array}{l}\text { Secondary } \\
\text { Roof Beam }\end{array}$ & $\begin{array}{l}\text { Secondary } \\
\text { Beams body }\end{array}$ & L40.40.4 & 12 & 3 \\
\hline $\begin{array}{c}\text { - Main } \\
\text { Beam joint }\end{array}$ & $\begin{array}{l}\text { Main Beams } \\
\text { body }\end{array}$ & L40.40.4 & 12 & 3 \\
\hline $\begin{array}{l}\text { Main Stairs } \\
\text { beam - }\end{array}$ & $\begin{array}{l}\text { Main Beams } \\
\text { Stairs body }\end{array}$ & L40.40.4 & 8 & 2 \\
\hline $\begin{array}{l}\text { Fulcrum } \\
\text { Stairs } \\
\text { Beam joint }\end{array}$ & $\begin{array}{c}\text { Fulcrum Stairs } \\
\text { Beam body }\end{array}$ & L40.40.4 & 8 & 2 \\
\hline $\begin{array}{l}\text { Fulcrum } \\
\text { Stairs }\end{array}$ & $\begin{array}{c}\text { Fulcrum Stairs } \\
\text { Beam body }\end{array}$ & L40.40.4 & 8 & 2 \\
\hline $\begin{array}{l}\text { Beam - } \\
\text { Columns } \\
\text { joint }\end{array}$ & Column Wing & L40.40.4 & 8 & 2 \\
\hline
\end{tabular}

BJ bolt 50, fu $=500 \mathrm{MPa}=5000 \mathrm{~kg} / \mathrm{cm}^{2}$

Table 11. Connection Planning Recap

\section{CONCLUSIONS AND SUGGESTION}

\section{Conclusion}

From the calculation and analysis has been done, it can be concluded as follows:

1. The results of calculation of secondary structure:

a. Planning for the roof and floor slab hospitals use floor deck of PT. Lysaght Bondek with $0.75 \mathrm{~mm}$ thick and $90 \mathrm{~mm}$ thick concrete slab and negative reinforcement are installed 10-200 for roof plate and negative reinforcement for floor slabs 12-250. $\varnothing$

b. Dimensions of roof joists with spans of $7.2 \mathrm{~m}$ on the roof using WF profile $300 \times 150$ $\times 6.5 \times 9$ and on the floor with the original profile WF $300 \times 200 \times 8 \times 14$, then converted into a castellated beam profile octogonal $442.5 \times 200 \times 8 \times 14$. 
Volume 03 Number 02 Maret 2020

c. Dimensions of the lift beam using WF profile $400 \times 200 \times 8 \times 13$.

d. Thick plates used $4 \mathrm{~mm}$ stairs and risers angled stiffener dimensions $65 \times 65 \times 8$.

e. Landing slab thickness used $9 \mathrm{~mm}$ and the dimensions of the beam landing using WF profile $100 \times 50 \times 5 \times 7$.

f. Dimensions main stairs beam and fulcrum stairs beam using WF profile $250 \times 125 \mathrm{x}$ $5 \times 8$.

2. The results of calculation of primary structure:
a. Dimensions of the transverse roof beam using octogonal profile castellated beam $427.5 \times 200 \times 8 \times 12$ and longitudinal roof beam using octogonal profile castellated beam $570 \times 200 \times 8 \times 13$.
b. Dimensions of the transverse floor beam using castellated beam profile octogonal profile $427.5 \times 200 \times 8 \times 12$ and beam elongated floor using octogonal profile castellated beam $617.5 \times 300 \times 10 \times 15$.
c. 1-4 floor column dimensions using a profile King Cross $588 \times 300 \times 12 \times 20$ dimension columns 5-7 floor using a profile King Cross 450 × $200 \times 9 \times 14$.
d. Used steel plate shear wall with $3 \mathrm{~mm}$ thick

\section{Suggestion}

Based on the description and discussion in the previous chapter, it can be given suggestions as follows:

a. In order to do the analysis and recalculation control well for the secondary structure, primer and calculation of connections. If required the calculation of the foundation.

b. Need for analysis of rigidity in the structure of the building.

c. It is expected that the need for a study to learn about the calculation of castellated beams octagonal also planning to use a steel plate shear wall (steel plate shear wall) more deeply by considering various things about the technical, economic and aesthetic.

\section{REFERENCES}

Hutabarat, Sarah Yosephin and Syahrizal. 2017. Analysis of economy Against Usage Castelatted Profile As Beams On Steel Construction (Case Study: BMW Showroom Development Project on Medan). Civil Engineering Journal. North Sumatra: Department of Civil Engineering Faculty of Engineering, University of North Sumatra. ISBN: 979-8382-51-X. Steel Structure I. 1997. Jakarta: Gunadarma, 1997.

National Standardization Agency. 2012. ISO 1726: 2012 Earthquake Resilience Planning Procedures For Non Structural Building And Building. Jakarta: BSN.

National Standardization Agency. 2013. ISO 1727: 2013 Minimum Expense For Designing Building And Other Structures. Jakarta: BSN.

National Standardization Agency. 2015. ISO 1729: 2015 Specification for Structural Steel Buildings. Jakarta: BSN.

Public Works Department. Regulations 1987. Imposition of Indonesia For Building (PPIUG 1987). Bandung: Directorate General of Human Settlements.

Purboningtyas, Andira Sari. 2016. Modification of Buildings Structural Design Santika Hotel on Bekasi Using King Cross Column and octagonal castellated beam. Thesis. 
IJTI (International Journal Of Transparantion Infrastructure) Available Online @http://jurnal.narotama.ac.id/index.php/ijti ISSN: 2597-4769 (ONLINE) ISSN : 2597-4734 (CETAK)

Volume 03 Number 02 Maret 2020

Surabaya: Faculty of Civil Engineering and Planning Sepuluh Nopember Institute of Technology Surabaya.

Sabelli, R. and Bruneaue, M. 2006. Steel Plate Shear Walls. American Institute of Steel Construction. Inc.

Seilie and Hooper. 2005 Steel Plate Shear Walls: Practical Design and Construction. The Steel Conference, April 2005.

Yulianti, Dewi. 2016. Study of Usage Profiles Steel with the Profile Hexagonal and Octogonal Castellated in Steel Building Structures. Thesis. Surabaya: Faculty of Civil Engineering and Planning Sepuluh Nopember Institute of Technology Surabaya. 